\title{
Prevalence of carbapenemase-producing organisms at the Kidney Center of Rawalpindi (Pakistan) and evaluation of an advanced molecular microarray-based carbapenemase assay
}

\author{
Sascha D Braun* $\star_{\ddagger 1}, 2$, Bushra Jamil ${ }^{\ddagger}{ }^{3}$, Muhammad A Syed ${ }^{4}$, Shahid A Abbasi ${ }^{5}$, Daniel \\ Wei $^{1,2}$, Peter Slickers ${ }^{1,2}$, Stefan Monecke ${ }^{1,2,6}$, Ines Engelmann ${ }^{1,2}$ \& Ralf Ehricht ${ }^{1,2}$ \\ ${ }^{1}$ Research \& Development, Abbott (Alere Technologies $\mathrm{GmbH}$ ), Jena, Germany \\ ${ }^{2}$ InfectoGnostics Research Campus, Jena, Germany \\ ${ }^{3}$ Department of Biogenetics, National University of Medical Sciences, Rawalpindi, Pakistan \\ ${ }^{4}$ Department of Microbiology, University of Haripur, Haripur, Pakistan \\ ${ }^{5}$ Department of Pathology, Al-Sayed Hospital (Pvt) Ltd, 1-Hill Park, Opp. Ayub Park, Jhelum Road, Rawalpindi, Pakistan \\ ${ }^{6}$ Technische Universität Dresden, Medizinische Fakultät "Carl Gustav Carus", Dresden, Germany \\ *Author for correspondence: Tel.: +49 3641311 1207; sascha.braun@alere.com \\ ${ }_{\ddagger}^{\ddagger}$ Authors contributed equally
}

\begin{abstract}
Aim: A DNA microarray-based assay for the detection of antimicrobial resistance (AMR) genes was used to study carbapenemase-producing organisms at the Kidney Center of Rawalpindi, Pakistan. Methods: The evaluation of this assay was performed using 97 reference strains with confirmed AMR genes. Testing of 7857 clinical samples identified 425 Gram-negative bacteria out of which 82 appeared carbapenem resistant. These isolates were analyzed using VITEK-2 for phenotyping and the described AMR assay for genotyping. Results: The most prevalent carbapenemase gene was blaNDM and in 12 isolates we detected two carbapenemase genes (e.g., blaNDM/blaOXA-48). Conclusion: Our prevalence data from Pakistan show that - as in other parts of the world - carbapenemase-producing organisms with different underlying resistance mechanisms are emerging, and this warrants intensified and constant surveillance.
\end{abstract}

First draft submitted: 13 March 2018; Accepted for publication: 30 May 2018; Published online: 25 June 2018

Keywords: carbapenemases $\bullet$ CarbDetect $\bullet \mathrm{CPO} \bullet \mathrm{ESBL} \bullet$ hospital $\bullet$ microarray $\bullet$ Pakistan

From the beginning of this century, extended-spectrum $\beta$-lactamases (ESBL) have been considered to be a major public health threat, as ESBLs can hydrolyze most cephalosporins and monobactams [1]. More than 700 types of ESBLs have been detected hitherto [2,3]. According to a recent study, the prevalence of ESBLs in Pakistan is alarmingly high, that is, above 80\% [4]. One remaining treatment option for ESBL-producing Enterobacteriaceae is the use of carbapenems (i.e., imipenem, ertapenem, meropenem and doripenem). However, the overuse of carbapenems to treat ESBL-positive pathogens has been selected for carbapenemase-producing and carbapenemresistant bacterial pathogens $[5,6]$. Carbapenemases are $\beta$-lactamases that can actively cleave the $\beta$-lactam ring of nearly all $\beta$-lactam antibiotics used so far in clinical practice. One notable exception is aztreonam, which is stable against metallo- $\beta$-lactamases [7].

Carbapenemase-producing organisms (CPOs) cause different types of infections including ventilator-associated pneumonia or urinary tract infections, which consequently are difficult to treat. Infections caused by CPOs are associated with higher in-hospital mortality (up to $70 \%$ ) related to inefficiency of primary therapeutic regimens $[4,8-$ 10].

During the last decade, CPOs that are resistant to almost all other antibiotics have emerged. CPOs expressing NDM-1 carbapenemase are a particular threat, as the blaNDM-1 gene is highly promiscuous, being readily transmittable between species and genera [11], with concomitant transfer of up to 14 different antibiotic resistance

Future $\because \%$ Medicine 
genes. There is a lot of evidence that CPOs with NDM-1 are prevalent in the population of the Indian subcontinent and that they are spreading, for example, via international travel $[6,12,13]$.

NDM-1 has been disseminated in different countries and is being reported globally. Because of its association with the Indian subcontinent, a number of studies have included samples from Pakistan, Sri Lanka, Bangladesh and India to evaluate the prevalence and spread of this enzyme. An overall prevalence of $18.5 \%$ blaNDM- 1 positive Enterobacteriaceae has been reported in 2011 for two military hospitals in Rawalpindi, Pakistan [14]. Another study conducted in two tertiary care hospitals in Pakistan from 2013 indicated prevalence rates of $23.0 \%$ for blaNDM-1, $25.0 \%$ for blaVIM and $1.5 \%$ for blaIMP [4].

Treatment options for CPOs are limited to polymyxins, tigecycline, aminoglycosides and fosfomycin [15]. Carbapenem resistance has instigated the use of tigecycline and colistin, and resistance has also been reported against these agents [16]. Until recently, all reported polymyxin resistance mechanisms were chromosomally mediated, involving modulation of two component regulatory systems $[17,18]$, and thus they were non-transferable. However, Liu et al. discovered in 2015 a plasmid-borne gene, $m c r-1$, which mediates resistance against colistin, and additional $m c r$ alleles have been described later on. It was also shown that $m c r$-associated resistance to colistin is transferable from cell to cell [19].

The CDC recommend active screening for CPOs [20-22]. Misidentification of carbapenem resistance could lead to inappropriate antibiotic treatment and dissemination of resistance [23]. There is an urgent need for diagnostic laboratories to introduce methods for a rapid molecular identification of carbapenemase genes in Gram-negative bacteria for infection control and prevention, surveillance, and for epidemiological purposes [5].

Current methods of detecting CPOs in patient samples, such as selective culture media followed by standard susceptibility testing (e.g., VITEK-2 (bioMerieux, Nürtingen, Germany), are time-consuming and do not provide genotyping data for surveillance and epidemiological questions. Therefore, several PCR-based assays have been developed that are able to detect carbapenemase genes directly in patient samples (e.g., Cepheids Xpert ${ }^{\circledR}$ Carba- $\mathrm{R}[18]$ (Cepheid, CA, USA), Check-Points Check-Direct CPE ${ }^{\circledR}$ test [24] (Check-Points, Wageningen, The Netherlands) or to identify carbapenemase genes in cultured bacterial isolates (e.g., Check-Points Check-MDR Carba [25]). However, a multitude of carbapenemase genes and their allelic variants are already known [26] and PCR-based tests normally cover only a small set of carbapenemase genes and alleles thereof.

The microarray-based assay presented herein provides a new option to simultaneously identify 43 globally important carbapenemase genes (including their allelic variants) and other important resistance genes (e.g., those encoding ESBLs, aminoglycoside or fluoroquinolone resistance) using isolated DNA from clonal bacterial culture.

We wanted to address two aims within this study. First, the overall performance of the microarray was to be assessed using genotypic and phenotypic profiles of reference strains and clinical isolates. Second, the newly evaluated array was to be used to study the prevalence of CPOs at the Kidney Center of Rawalpindi, Pakistan.

\section{Materials \& methods}

Evaluation of the CarbDetect AS-2 kit

The function of the CarbDetect AS-2 kit (Alere Technologies GmbH, Jena, Germany) was evaluated using a set of 97 genotypically characterized reference strains (Table 1, and Supplementary Table 1) from different sources (University Medical Center of Dresden, Dresden, Germany [ $\mathrm{n}=3]$, University Medical Center of Jena, Jena, Germany [ $=10]$, German Collection of Microorganisms and Cell Cultures, Braunschweig, Germany [DSMZ; n = 4], University of Fribourg, Fribourg, Switzerland [ $=72]$, in-house collection of Alere, Jena, Germany $[n=6]$ and the German National Reference Laboratory for Multidrug-Resistance Gram-negative Bacteria at Bochum University, Bochum, Germany [NRL, $\mathrm{n}=2])$. The investigated strain collection comprised Acinetobacter baylyi $(\mathrm{n}=1)$, Acinetobacter junii $(\mathrm{n}=1)$, Acinetobacter haemolyticus $(\mathrm{n}=1)$, Acinetobacter baumannii $(\mathrm{n}=20)$, Acinetobacter lwoffii $(\mathrm{n}=2)$, Chryseobacterium gleum $(\mathrm{n}=1)$, Chryseobacterium meningosepticum $(\mathrm{n}=1)$, Chryseobacterium indologenes $(\mathrm{n}=4)$, Citrobacter freundii $(\mathrm{n}=3)$, Enterobacter aerogenes $(\mathrm{n}=1)$, Enterobacter cloacae $(\mathrm{n}=2)$, Escherichia coli $(\mathrm{n}=14)$, Klebsiella oxytoca $(\mathrm{n}=1)$, Klebsiella pneumoniae $(\mathrm{n}=13)$, Proteus mirabilis $(\mathrm{n}=2)$, Pseudomonas aeruginosa $(\mathrm{n}=14)$, Pseudomonas fluorescens $(\mathrm{n}=3)$, Pseudomonas putida $(\mathrm{n}=1)$, Pseudomonas stutzeri $(\mathrm{n}=1)$, Ralstonia pickettii $(\mathrm{n}=1)$, Salmonella enterica $(\mathrm{n}=5)$, Serratia marcescens $(\mathrm{n}=2)$, Shewanella algae $(\mathrm{n}=1)$, Shewanella oneidensis $(\mathrm{n}=1)$ and Shigella boydii $(\mathrm{n}=1)$. All reference strains were used only to analyze confirmed antimicrobial resistance (AMR) genes, which were detected by others methods than microarray (i.e., PCR or sequencing). These confirmed AMR genes are listed in Table 1 . Reference strains were analyzed by microarray using the same procedures as described in 
Table 1. Evaluation of the CarbDetect AS-2 kit: competitive study using reference strains with confirmed antimicrobial

\section{resistance genes.}

\begin{tabular}{|c|c|c|c|c|}
\hline Reference strain number & Species & Confirmed AMR genes & Detected AMR genes by microarray & Concordance in \% \\
\hline 278787 & Acinetobacter baylyi & blaPER-2 & blaPER-2 & 100 \\
\hline 278788 & Acinetobacter junii & blaPER-2 & blaPER-2 & 100 \\
\hline 242273 & Acinetobacter haemolyticus & blaOXA-58, blaOXA-214 & blaOXA-58, blaOXA-214 & 100 \\
\hline 208268 & Acinetobacter baumannii & blaOXA-72 (blaOXA-40-like) & blaOXA-40-like & 100 \\
\hline 219847 & A. baumannii & blaGIM & blaGIM (consensus) & 100 \\
\hline 240618 & A. baumannii & blaOXA-40 & blaOXA-40-like & 100 \\
\hline 240734 & A. baumannii & blaOXA-97 (blaOXA-58-like) & bla-OXA-58-like & 100 \\
\hline 240736 & A. baumannii & blaOXA-143 & blaOXA-143/182/253/255 & 100 \\
\hline 240737 & A. baumannii & blaNDM-2, aadB, ant2 & blaNDM (consensus), aadB, ant2 & 100 \\
\hline 240741 & A. baumannii & blaGES-14 & blaGES (consensus) & 100 \\
\hline 240742 & A. baumannii & blaGES-11, blaOXA-23 & blaGES (consensus), blaOXA-23 & 100 \\
\hline 240770 & A. baumannii & $\begin{array}{l}\text { blaNDM, blaOXA-23, blaOXA-69 } \\
\text { (blaOXA-51-like) }\end{array}$ & $\begin{array}{l}\text { blaNDM (consensus), blaOXA-23, } \\
\text { blaOXA-51-like }\end{array}$ & 100 \\
\hline 240785 & A. baumannii & blaOXA-23, blaPER-1, aadA1, aphA & blaOXA-23-like, blaPER-1, aadA1, aphA & 100 \\
\hline 240786 & A. baumannii & blaOXA-23, blaPER-7 & blaOXA-23-like, blaPER-1 & 100 \\
\hline 240787 & A. baumannii & blaOXA-23, blaGES-11 & blaOXA-23-like, blaGES-11 & 100 \\
\hline 240788 & A. baumannii & blaOXA-24 (b/aOXA-40-like) & blaOXA-40-like & 100 \\
\hline 240789 & A. baumannii & blaOXA-51 & blaOXA-51-like & 100 \\
\hline 240794 & A. baumannii & $\begin{array}{l}\text { blaOXA-69 (blaOXA-51-like), } \\
\text { blaVEB-1, blaOXA-10 }\end{array}$ & $\begin{array}{l}\text { blaOXA-51-like, blaVEB (consensus), } \\
\text { blaOXA-10 }\end{array}$ & 100 \\
\hline 274215 & A. baumannii & $\begin{array}{l}\text { blaOXA-107 (blaOXA-51-like), } \\
\text { ISABa1 }\end{array}$ & blaOXA-51-like, ISABa1 & 100 \\
\hline 274216 & A. baumannii & blaOXA-64 (blaOXA-51-like), blaACT & blaOXA-51-like, blaACT & 100 \\
\hline 274217 & A. baumannii & blaOXA-201 (blaOXA-51-like) & blaOXA-51-like & 100 \\
\hline 274218 & A. baumannii & blaOXA-82 (blaOXA-51-like) & bla-OXA-51-like & 100 \\
\hline 278800 & A. baumannii & blaTMB-1 & blaTMB-1 & 100 \\
\hline 240796 & Acinetobacter Iwoffii & blaOXA-134 & blaOXA-134/235/284 & 100 \\
\hline 278794 & A. Iwoffii & blaOXA-134 & blaOXA-134/235/284 & 100 \\
\hline 242277 & Chryseobacterium gleum & blaIND (CBG-1) & blaIND (consensus) & 100 \\
\hline 240744 & $\begin{array}{l}\text { Chryseobacterium } \\
\text { meningoseptica }\end{array}$ & blaCME-2, blaGOB-1 & blaCME (consensus), blaGOB (consensus) & 100 \\
\hline 240766 & $\begin{array}{l}\text { Chryseobacterium } \\
\text { indologenes }\end{array}$ & blalND-1 & blalND (consensus) & 100 \\
\hline 240767 & C. indologenes & blaIND-2 & blalND (consensus) & 100 \\
\hline 240769 & C. indologenes & blalND-4 & blaIND (consensus) & 100 \\
\hline 248612 & C. indologenes & blaIND-3 & blalND (consensus) & 100 \\
\hline 240802 & Citrobacter freundii & blaVEB-1, qnrB & blaVEB (consensus), qnrB & 100 \\
\hline 242269 & C. freundii & $\begin{array}{l}\text { blaVIM-2, blaTEM-1, blaOXA-9, } \\
\text { blaOXA-10 }\end{array}$ & $\begin{array}{l}\text { blaVIM (consensus), blaTEM (consensus), } \\
\text { blaOXA-9, blaOXA-10 }\end{array}$ & 100 \\
\hline 242274 & C. freundii & $\begin{array}{l}\text { blaNDM-1, blaTEM, blaCTX-M15, } \\
\text { blaOXA-1, blaOXA-9, blaOXA-10, } \\
\text { armA, dfrA12, aadA2 }\end{array}$ & $\begin{array}{l}\text { blaNDM (consensus), blaTEM (consensus), } \\
\text { blaCTX-M1/15, blaOXA-9, blaOXA-10, } \\
\text { armA, dfrA12, aadA2 }\end{array}$ & 100 \\
\hline 242279 & Enterobacter aerogenes & qnrA1 & qnrA1 & 100 \\
\hline 240750 & Enterobacter cloacae & $n m c A$, blaMIR & blalMI-3 (NmcA), blalMI-R, blaMIR & 100 \\
\hline 278791 & E. cloacae & $\begin{array}{l}\text { blaOXA-163 (blaOXA-48-like), dfrA1, } \\
\text { sul1, qnrB }\end{array}$ & blaOXA-48-like, dfrA1, sul1, qnrB & 100 \\
\hline 157048 & Escherichia coli & blaOXA-45 & blaOXA-45 & 100 \\
\hline 174396 & E. coli & $\operatorname{aac}\left(3^{\prime}\right)-I V a$ & $\operatorname{aac}\left(3^{\prime}\right)-I V a$ & 100 \\
\hline 187734 & E. coli & qnrC, dfrA13 & qnrC, dfrA13 & 100 \\
\hline 204512 & E. coli & blaDHA & blaDHA & 100 \\
\hline
\end{tabular}


Table 1. Evaluation of the CarbDetect AS-2 kit: competitive study using reference strains with confirmed antimicrobial resistance genes (cont.).

\begin{tabular}{|c|c|c|c|c|}
\hline Reference strain number & Species & Confirmed AMR genes & Detected AMR genes by microarray & Concordance in \% \\
\hline 208263 & E. coli & blaNDM, blaCTX-M1, rmtC & blaNDM (consensus), blaCTX-M1/15, rmtC & 100 \\
\hline 208270 & E. coli & blaOXA-2 & blaOXA-2 (consensus) & 100 \\
\hline 240614 & E. coli & blalMP-1, aac( $\left.6^{\prime}\right)$-lic, dfrA5, sul2 & blaIMP (consensus), aac $\left(6^{\prime}\right)$-lic & 100 \\
\hline 240615 & E. coli & blaKPC-2, aadA4 & blaKPC (consensus), aadA4 & 100 \\
\hline 240772 & E. coli & $\begin{array}{l}\text { blaNDM-1, blaTEM, blaOXA-1, } \\
\operatorname{aadA2,~aacC2,~aacA4,~dfrA17,~sul2,~} \\
\text { mph (consensus), mrx (consensus) }\end{array}$ & $\begin{array}{l}\text { blaNDM (consensus), blaTEM (consensus), } \\
\text { blaOXA-1, aadA2, aacC2, aacA4, dfrA17, } \\
\text { sul2, mph (consensus), mrx (consensus) }\end{array}$ & 100 \\
\hline 240773 & E. coli & blaNDM-4, blaCTX-M1, blaCMY-6 & $\begin{array}{l}\text { blaNDM (consensus), blaCTX-M1/15, } \\
\text { blaCMY (consensus) }\end{array}$ & 100 \\
\hline 242280 & E. coli & qnrA1 & qnrA1 & 100 \\
\hline 248610 & E. coli & blaOXA-204 (blaOXA-48-like) & blaOXA-48-like & 100 \\
\hline 278798 & E. coli & $\operatorname{aac}\left(6^{\prime}\right)-I b-c r, d f r A 7$ & $\operatorname{aac}\left(6^{\prime}\right)-I b, d f r A 7$ & 50 \\
\hline 278799 & E. coli & blaCTX-M9-like (blaCTX-M14), qepA & blaCTX-M9, qepA & 100 \\
\hline 279601 & Klebsiella oxytoca & blaVIM, blaACC & blaVIM (consensus), blaACC & 100 \\
\hline 208269 & Klebsiella pneumoniae & blaNDM, blaCMY & blaNDM (consensus), blaCMY (consensus) & 100 \\
\hline 215771 & K. pneumoniae & blaOXA-181 & blaOXA-181/232 & 100 \\
\hline 215792 & K. pneumoniae & blaOXA-9, dfrA14, sul3 & blaOXA-9, dfrA14, sul3 & 100 \\
\hline 240610 & K. pneumoniae & blaOXA-48 & blaOXA-48-like & 100 \\
\hline 240612 & K. pneumoniae & blaVIM-1 & blaVIM (consensus) & 100 \\
\hline 240616 & K. pneumoniae & blaKPC-2, blaCTX-M2, dfrA15, sul2 & $\begin{array}{l}\text { blaKPC (consensus), blaCTX-M2, dfrA15, } \\
\text { sul2 }\end{array}$ & 100 \\
\hline 240749 & K. pneumoniae & $\begin{array}{l}\text { blaKPC-3, blaCTX-M15, blaTEM-1, } \\
\text { blaSHV-11 }\end{array}$ & $\begin{array}{l}\text { blaKPC (consensus), } \\
\text { blaCTX-blablaCTX-M1/15, blaTEM } \\
\text { (consensus), blaSHV (consensus) }\end{array}$ & 100 \\
\hline 240757 & K. pneumoniae & blaIMP-8, blaSHV-12, dfrA19, sul2 & $\begin{array}{l}\text { blalMP (consensus), blaSHV (consensus), } \\
\text { dfrA19, sul2 }\end{array}$ & 100 \\
\hline 240781 & K. pneumoniae & $\begin{array}{l}\text { blaVIM-4, blaTEM-1, blaCTX-M1/15, } \\
\text { qnrs }\end{array}$ & $\begin{array}{l}\text { blaVIM (consensus), blaTEM (consensus), } \\
\text { blaCTX-M1/15, qnrs }\end{array}$ & 100 \\
\hline 240798 & K. pneumoniae & $\begin{array}{l}\text { blaOXA-232, blaSHV-1, blaTEM-1, } \\
\text { blaCTX-M1/15, blaOXA-1 }\end{array}$ & $\begin{array}{l}\text { blaOXA-181/232, blaSHV (consensus), } \\
\text { blaTEM (consensus), CTX-M1/15, } \\
\text { blaOXA-1 }\end{array}$ & 100 \\
\hline 240799 & K. pneumoniae & $\begin{array}{l}\text { blaOXA-232, blaNDM-1, blaSHV-1, } \\
\text { blaTEM-1, blaCTX-M1/15, blaOXA-1, } \\
\text { dfrA1, dfrA14, sul1, sul2 }\end{array}$ & $\begin{array}{l}\text { blaOXA-181/232, blaNDM (consensus), } \\
\text { blaSHV (consensus), blaTEM (consensus), } \\
\text { blaCTX-M1/15, blaOXA-1 }\end{array}$ & 100 \\
\hline 242275 & K. pneumoniae & blaOXA-162 (blaOXA-48-like) & blaOXA-48-like & 100 \\
\hline 280640 & K. pneumoniae & $r m t B$ & $r m t B$ & 100 \\
\hline 240777 & Proteus mirabilis & $\begin{array}{l}\text { blaNDM-1, blaCMY-16, blaOXA-10, } \\
\operatorname{armA}\end{array}$ & $\begin{array}{l}\text { blaNDM (consensus), blaCMY (consensus), } \\
\text { blaOXA-10 (consensus), armA }\end{array}$ & 100 \\
\hline 242267 & P. mirabilis & blaVEB-6, blaNDM-1, qnrD & $\begin{array}{l}\text { blaVEB (consensus), blaNDM (consensus), } \\
\text { qnrD }\end{array}$ & 100 \\
\hline 204517 & Pseudomonas aeruginosa & blaVIM-1, $\operatorname{aac}\left(3^{\prime}\right)-I c$ & blaVIM (consensus), $\operatorname{aac}\left(3^{\prime}\right)-I c$ & 100 \\
\hline 227120 & $P$. aeruginosa & blalMP-31, $\operatorname{aac}\left(3^{\prime}\right)-I c$ & blalMP (consensus), $\operatorname{aac}\left(3^{\prime}\right)-/ c$ & 100 \\
\hline 227126 & $P$. aeruginosa & blalMP-2 & blalMP (consensus) & 100 \\
\hline 240613 & $P$. aeruginosa & blaVIM-2 & blaVIM (consensus) & 100 \\
\hline 240617 & $P$. aeruginosa & blaIMP-1, $\operatorname{aac}\left(6^{\prime}\right)-31$ & blaIMP (consensus), aac $\left(6^{\prime}\right)-31$ & 100 \\
\hline 240740 & $P$. aeruginosa & blaGES-2, aac(3')-la & blaGES (consensus), aac $\left(3^{\prime}\right)-l a$ & 100 \\
\hline 240760 & P. aeruginosa & blalMP-13 & blaIMP (consensus) & 100 \\
\hline 240761 & $P$. aeruginosa & blalMP-15 & blaIMP (consensus) & 100 \\
\hline 240762 & $P$. aeruginosa & blaIMP-19 & blalMP (consensus) & 100 \\
\hline 240783 & $P$. aeruginosa & $\begin{array}{l}\operatorname{aac}\left(6^{\prime}\right)-l b, \text { blaVIM-2, blaPER-1, strA, } \\
\operatorname{str} B\end{array}$ & $\operatorname{aac}\left(6^{\prime}\right)-l b, b / a \mathrm{VIM}-2$, blaPER-1, strA, strB & 100 \\
\hline
\end{tabular}


Table 1. Evaluation of the CarbDetect AS-2 kit: competitive study using reference strains with confirmed antimicrobial resistance genes (cont.).

\begin{tabular}{|c|c|c|c|c|}
\hline Reference strain number & Species & Confirmed AMR genes & Detected AMR genes by microarray & Concordance in \% \\
\hline 242278 & P. aeruginosa & blaSPM-1, rmtD & blaSPM-1, rmtD & 100 \\
\hline 248608 & $P$. aeruginosa & blalMP-29 & blalMP (consensus) & 100 \\
\hline 278789 & P. aeruginosa & blaGES-1, aac(3')-lb, aac( $\left.6^{\prime}\right)-\mathrm{lb}$ & blaGES (consensus), $\operatorname{aac}\left(3^{\prime}\right)-1 b, \operatorname{aac}\left(6^{\prime}\right)-l b$ & 100 \\
\hline 278792 & P. aeruginosa & blaOXA-18 & blaOXA-18 & 100 \\
\hline 202104 & Pseudomonas fluorescens & $r m t A$ & $r m t A$ & 100 \\
\hline 248793 & P. fluorescens & blaBIC-1 & blaBIC-1 & 100 \\
\hline 278793 & P. fluorescens & blaBIC-1 & blaBIC-1 & 100 \\
\hline 240784 & Pseudomonas putida & blaVIM-5 & blaVIM (consensus) & 100 \\
\hline 278797 & Pseudomonas stutzeri & blaDIM-1 & blaDIM (consensus) & 100 \\
\hline 240801 & Ralstonia pickettii & blaOXA-60 & blaOXA-60 & 100 \\
\hline 240764 & Salmonella enterica & blalMP-4 & blalMP (consensus) & 100 \\
\hline 254995 & S. enterica & grm & grm & 100 \\
\hline 255023 & S. enterica & $\operatorname{aac}\left(3^{\prime}\right)-l e$ & $\operatorname{aac}\left(3^{\prime}\right)-l e$ & 100 \\
\hline 278790 & S. enterica & blaPER-1 & blaPER-1 & 100 \\
\hline 281793 & S. enterica & blaCTX-M8 & blaCTX-M8 & 100 \\
\hline 240751 & Serratia marcescens & blaSME-1 & blaSME (consensus) & 100 \\
\hline 240765 & S. marcescens & blalMP-11 & blalMP (consensus) & 100 \\
\hline 240791 & Shewanella algae & blaOXA-55, qnrA3 & blaOXA-55 & 100 \\
\hline 278796 & Shewanella oneidensis & blaOXA-54 & blaOXA-54 & 100 \\
\hline 227453 & Shigella boydii & blaCTX-M2 & blaCTX-M2 & 100 \\
\hline n.a. & & & $\operatorname{aac}\left(6^{\prime}\right)-I I$ & n.d. \\
\hline n.a. & & & $\operatorname{aac}\left(6^{\prime}\right)-I l a$ & n.d. \\
\hline n.a. & & & aac-aph & n.d. \\
\hline n.a. & & & blaFOX & n.d. \\
\hline n.a. & & & blaKHM (consensus) & n.d. \\
\hline n.a. & & & blaMOX (consensus) & n.d. \\
\hline n.a. & & & blaMOX-CMY9 & n.d. \\
\hline n.a. & & & blaOXA-279 & n.d. \\
\hline n.a. & & & blaOXA-292 & n.d. \\
\hline n.a. & & & blaPAM-1 & n.d. \\
\hline n.a. & & & blaSFH-1 & n.d. \\
\hline n.a. & & & blaSMB-1 & n.d. \\
\hline n.a. & & & $n p m A$ & n.d. \\
\hline Overall concordance (mean) & & & & 98.8 \\
\hline
\end{tabular}

Braun et al. [27]. The concordance $\mathrm{C}$ was determined using following formula ( $\mathrm{n}=$ number):

$$
C=\frac{100 \cdot n_{\text {detected AMR-genes }}}{n_{\text {confirmed AMR-gene }}}
$$

A calculation of the true-positive and true-negative rate is difficult for the herein-described multiparameter assay (which detects 134 targets simultaneously), as the only reference method which could be used for that is whole genome sequencing. The costs to analyze all reference strains and clinical isolates using whole genome sequencing are far too high. Therefore, only strains with confirmed AMR genes were used to calculate the quality of the CarbDetect AS-2 kit. 
Clinical isolates: growth conditions, susceptibility testing \& genomic DNA extraction

We investigated non-duplicate, microbiological samples $(\mathrm{n}=7857)$ taken from patients admitted to the Kidney Center (Al-Sayed Hospital, with 150 beds), Rawalpindi, between January and May in 2016. The Kidney Center is a tertiary/quaternary care hospital that is mainly specialized in the treatment of renal ailments and in kidney, liver and bone marrow transplantations. Most of the patients included in the study suffered from chronic renal failure or received kidney transplants.

Out of all samples tested, 425 Gram-negative bacteria (i.e., Enterobacteriaceae and non-fermenter) were isolated. These isolates were subjected to susceptibility tests by the standard Kirby-Bauer disc diffusion method and those strains that were resistant to carbapenems were selected for further characterization. The sample sites from which carbapenem-resistant isolates were obtained included urine $(n=24)$, Foley catheter $(n=16)$, pus $(n=13)$, throat swab $(n=9)$, endotracheal tube $(n=7)$, bronchoalveolar lavage $(n=5)$, wound swab $(n=3)$, double-J stent $(n=2)$, blood culture $(n=1)$, central venous pressure tube $(n=1)$ and nasogastric tube $(n=1)$. Species identification of carbapenem-resistant isolates was performed using the API 20 E assay and VITEK-2 (both from bioMérieux, Nürtingen, Germany).

All carbapenem-resistant isolates were cultivated on tryptone yeast agar and subsequently retested using an automated microdilution technique (VITEK-2, card: AST-248, bioMérieux) that included the following antibiotics, imipenem, meropenem, cefepime, cefotaxime, ceftazidime, piperacillin, piperacillin/tazobactam, aztreonam, amikacin, gentamicin, tobramycin, ciprofloxacin, moxifloxacin, co-trimoxazole, tigecycline, fosfomycin and colistin. The interpretation of antimicrobial susceptibility tests was performed automatically by the VITEK-2 Expert software (bioMerieux, VITEK-2 system version: 07.01, MIC guidelines: EUCAST 2014 + CLSI 2014 D) according to the European Committee on Antimicrobial Susceptibility Testing (EUCAST) and/or Clinical and Laboratory Standards Institute (CLSI) breakpoints. All carbapenem-resistant isolates were additionally tested with a modified Hodge test [28] to identify isolates with overexpression of both AmpC $\beta$-lactamases in combination with porin loss and efflux pumps [29,30] using a $10 \mu \mathrm{g}$ meropenem susceptibility disk (Oxoid Ltd, Basingstoke, UK).

Genomic DNA was extracted using the Qiagen DNeasy Blood \& Tissue kit (Qiagen GmbH, Hilden, Germany) according to manufacturer's instructions. When necessary, DNA was concentrated to at least $100 \mathrm{ng} / \mu \mathrm{l}$ using a SpeedVac centrifuge (Eppendorf, Hamburg, Germany) at room temperature for $30 \mathrm{~min} / 1400$ r.p.m. Recovered genomic DNA $(5 \mu \mathrm{l})$ was used directly for internal biotin labeling and subsequent hybridization.

\section{Clinical isolates: antimicrobial resistance genotyping}

For all isolates, the carbapenemase, ESBL, narrow-spectrum $\beta$-lactamase (NSBL), amp $C$, aminoglycoside, macrolide, fluoroquinolone and co-trimoxazole genotypes were detected using the CarbDetect AS-2 kit. The data were automatically summarized by the 'result collector', a software tool developed by Abbott (Alere Technologies $\mathrm{GmbH}$ ). Detailed results to all detected AMR genes are given in the Supplementary Tables 2 and 3. The $m c r-1$ and mcr-2 genes, mediating colistin resistance, were determined by PCR [19]. No genetic markers for tigecycline and fosfomycin resistance were included because resistance to these compounds is related to mutations in ubiquitous genes rather than to an acquisition of dedicated genes [31].

An AMR-genotype was defined as a group of genes that have been described to confer resistance to a family of antibiotics (e.g., the AMR-genotype 'blaNDM' confers resistance to all tested $\beta$-lactam antibiotics or 'aac6, aac6ib, aadAl' confer resistance to aminoglycosides).

The concordance described in Table 3 was calculated between the resistance pattern as predicted by the genotypic profile and the actual phenotypic profile as determined by VITEK. For a better comparison between the different AMR families, a mean concordance within each family was also calculated. An overall concordance was calculated as a mean to all calculated values.

\section{Multiplex labeling, hybridization \& data analysis}

Array experiments were performed using the CarbDetect AS-2 kit. This test is an advanced version of the original CarbDetect AS-1 kit (Abbott [Alere Technologies GmbH], Jena, Germany). Some of the hybridization probes and labeling primers have been described previously [27]. In a direct comparison between both kits, 104 new target genes could now be detected with the advanced version. All target genes of the CarbDetect AS-2 kit and the differences to the original AS-1 kit are listed in Supplementary Table 4. 


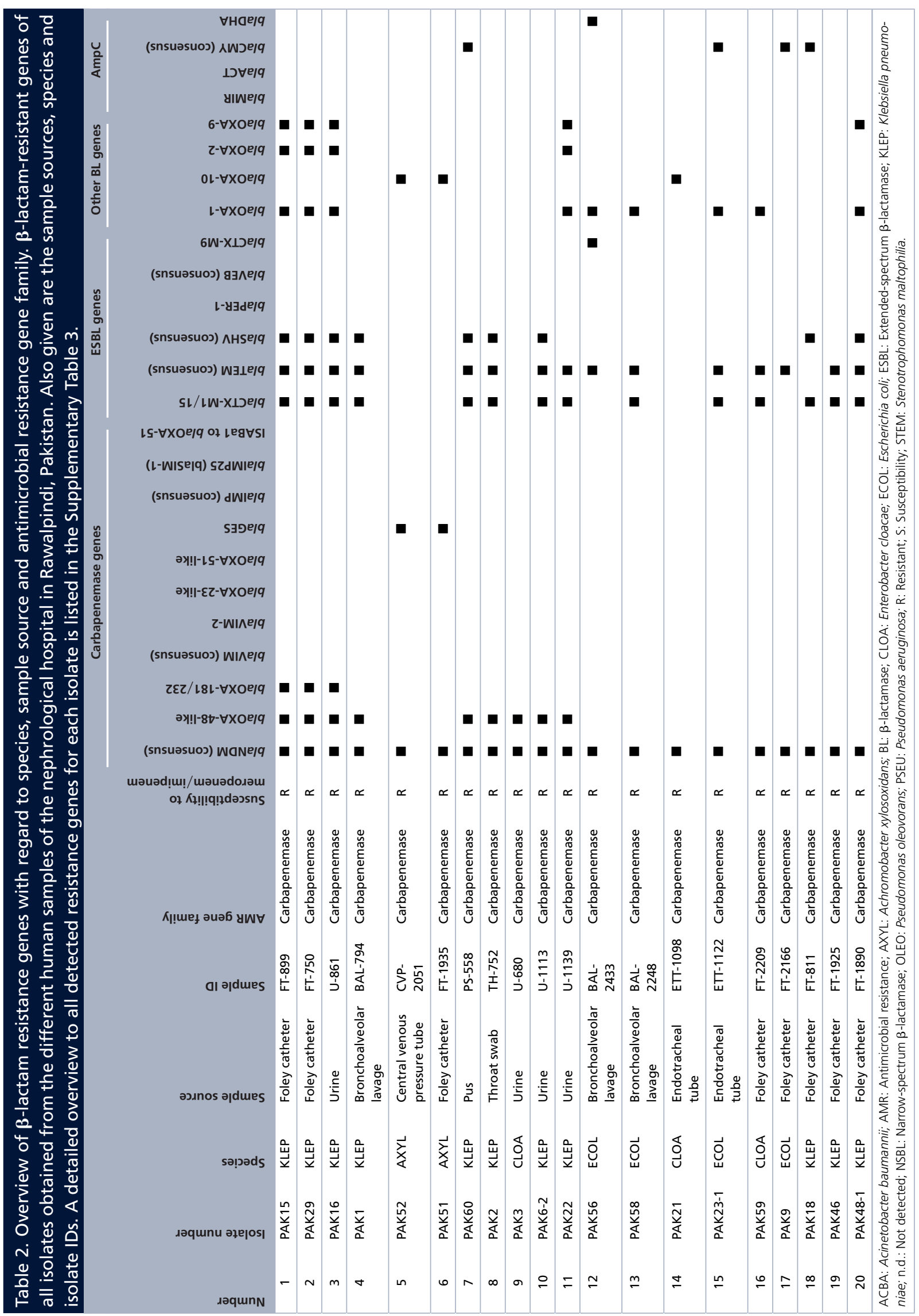




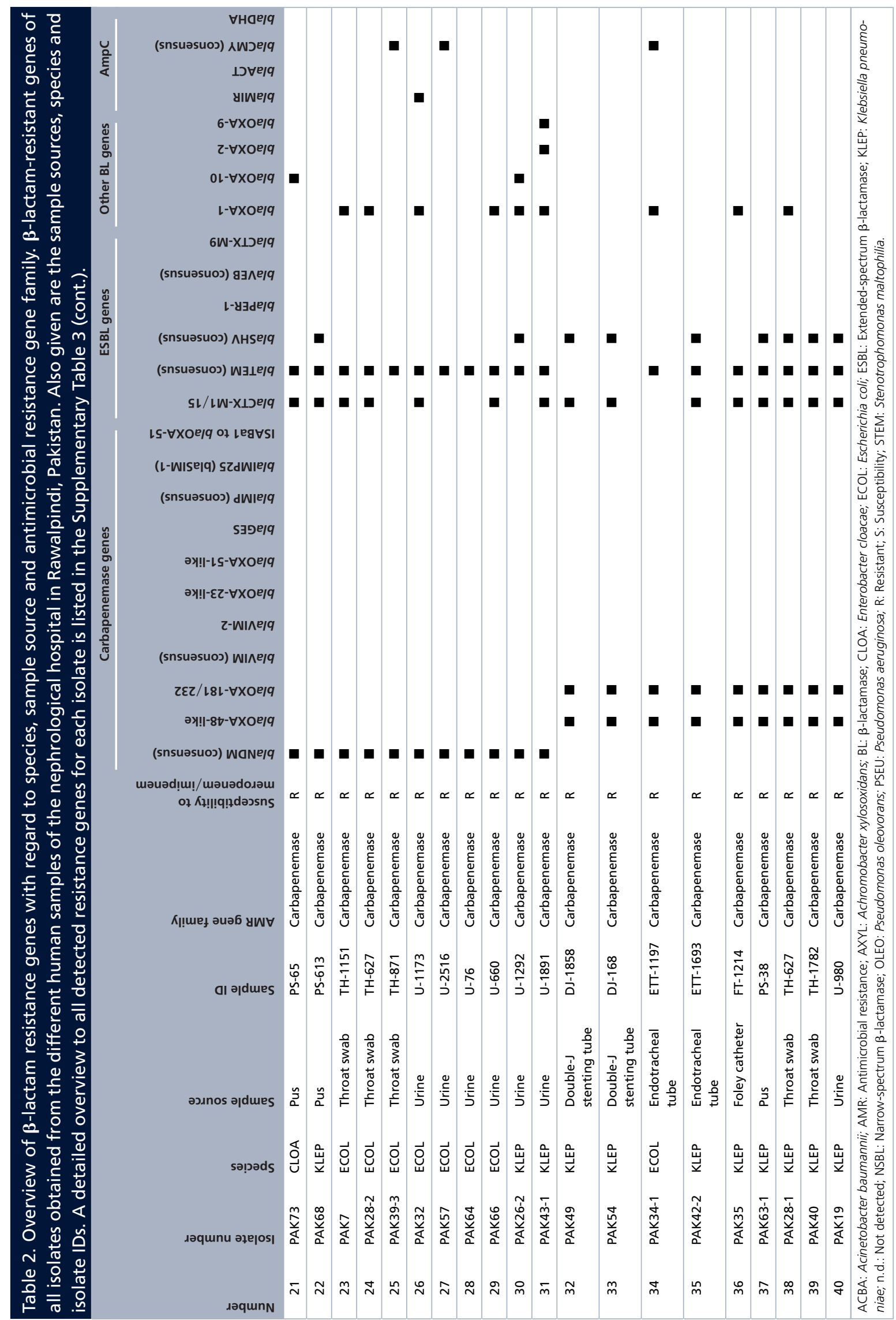




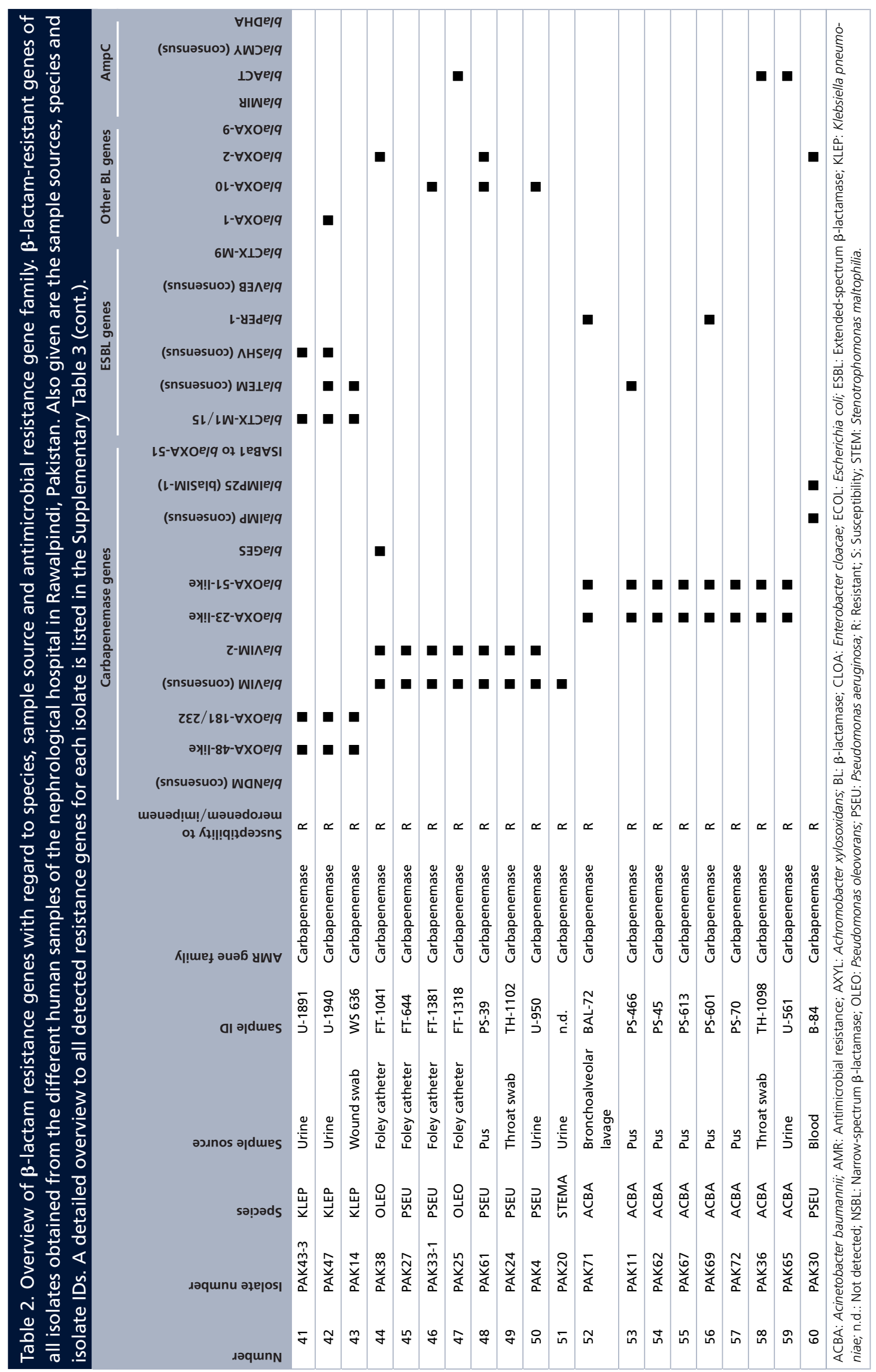




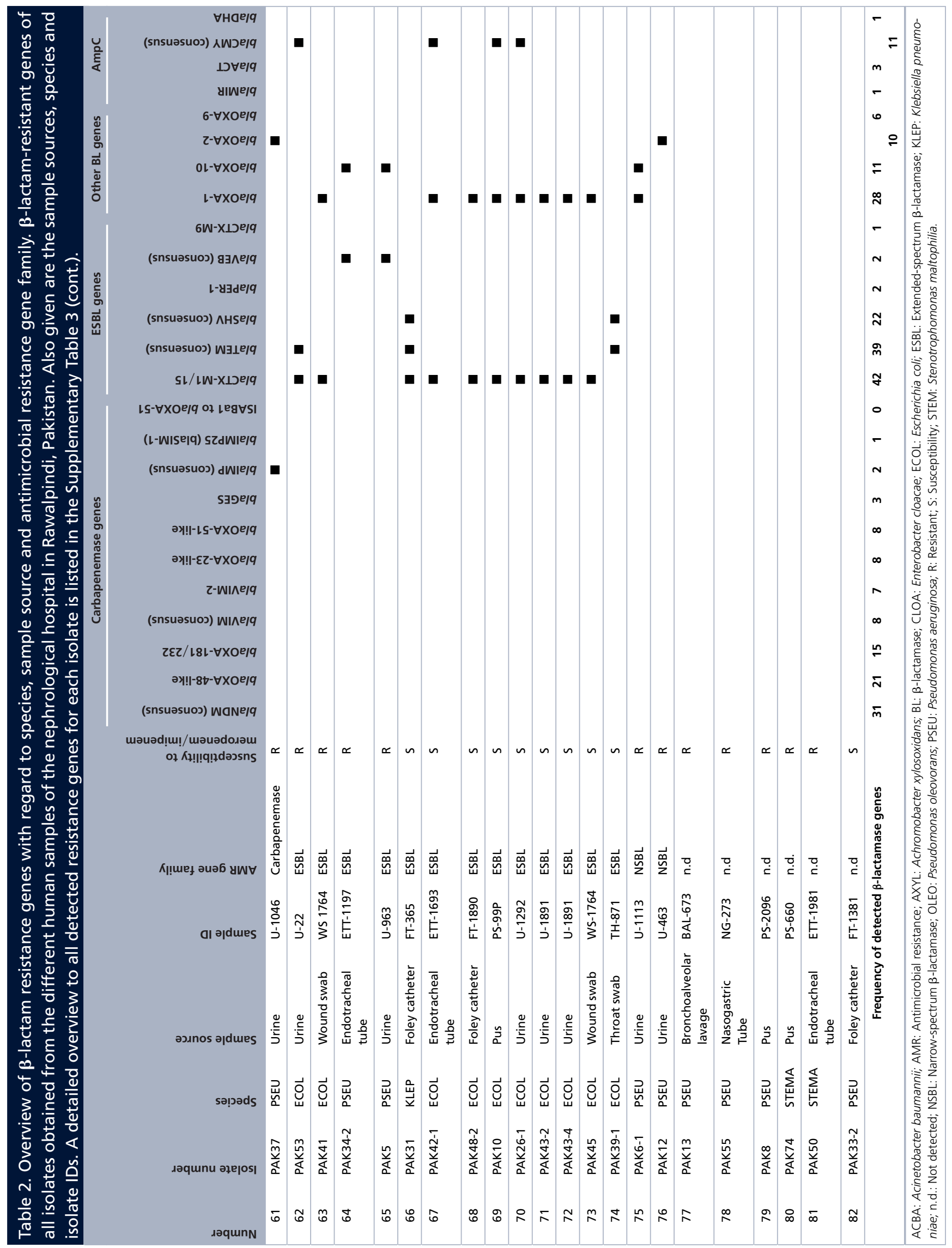




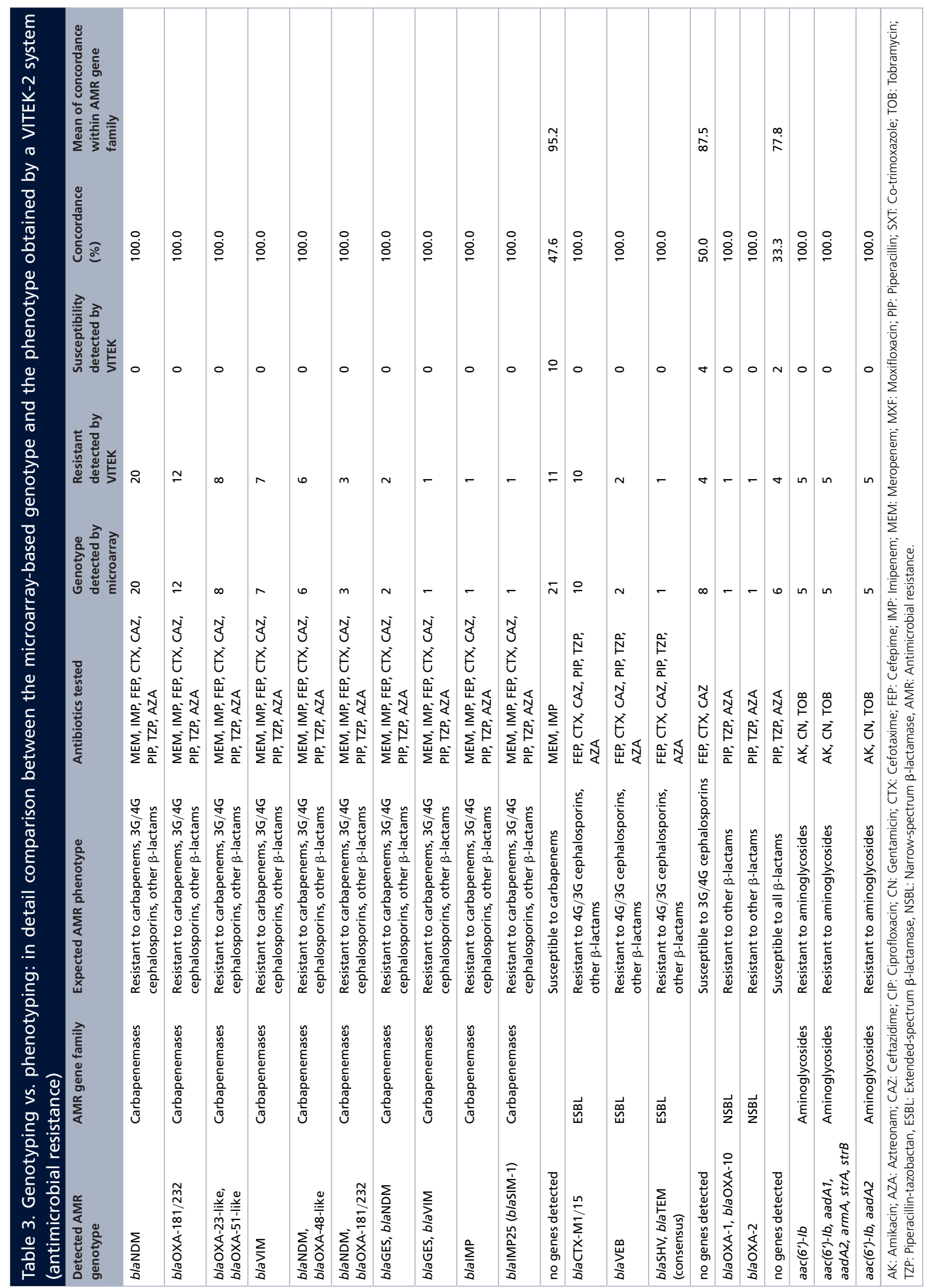




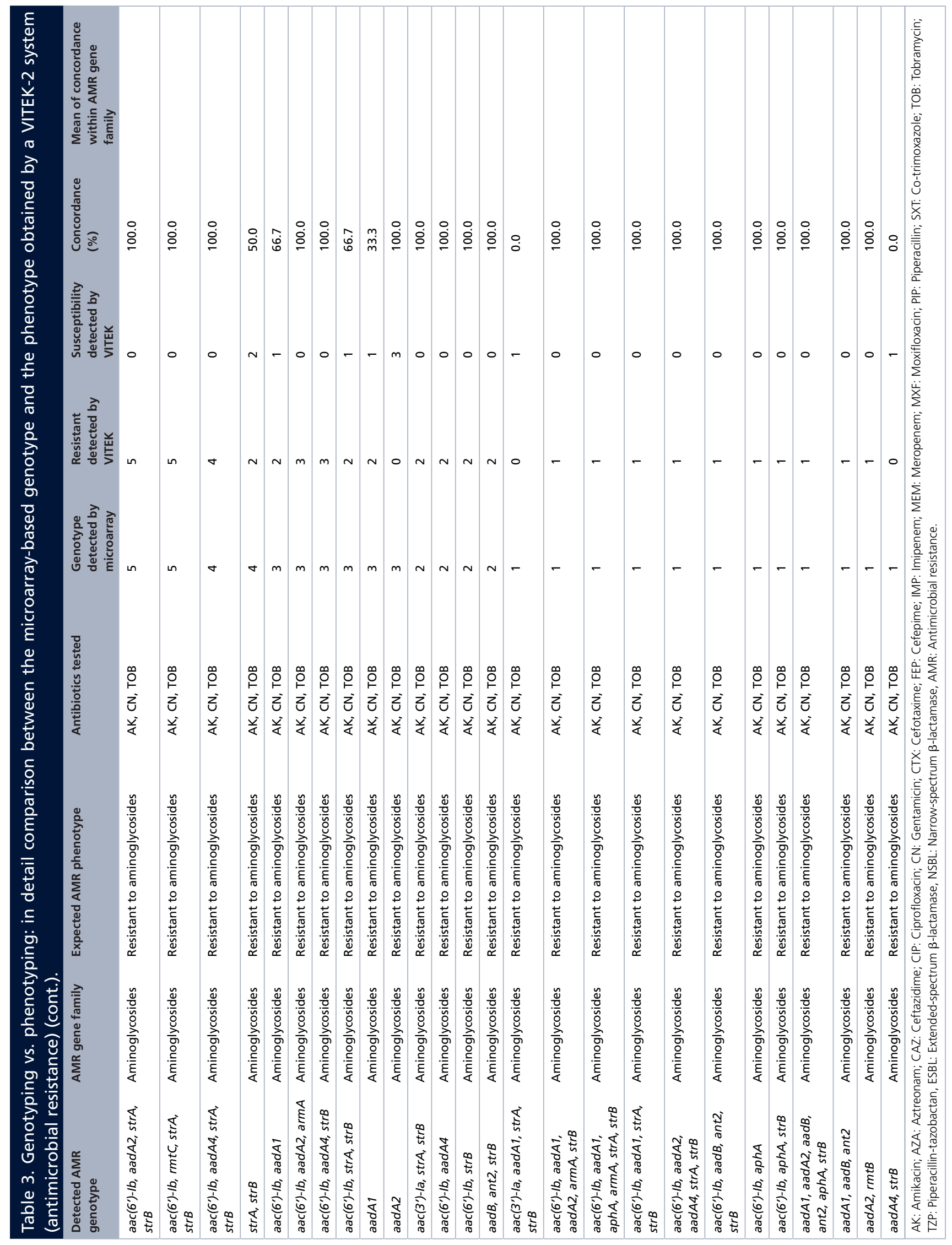




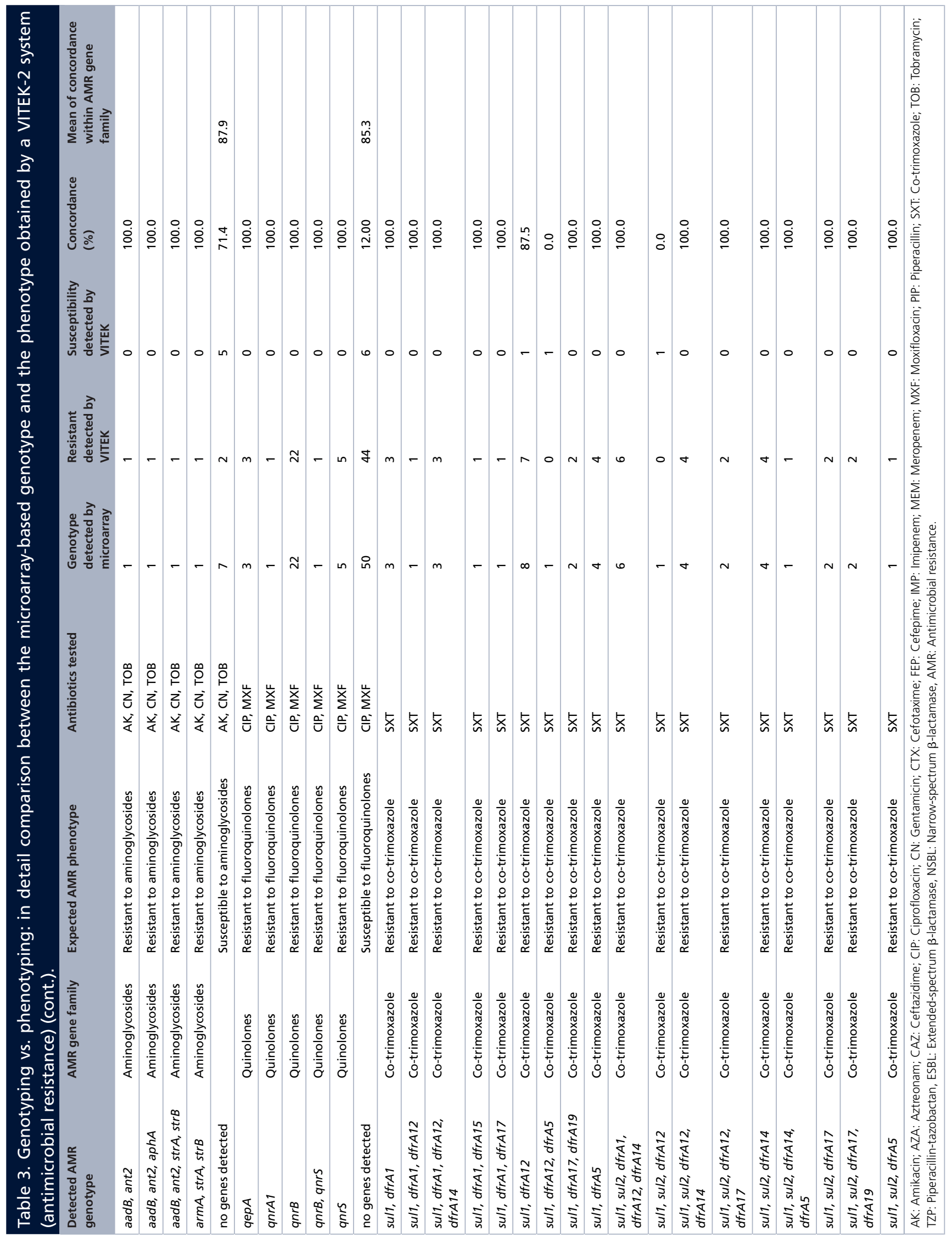




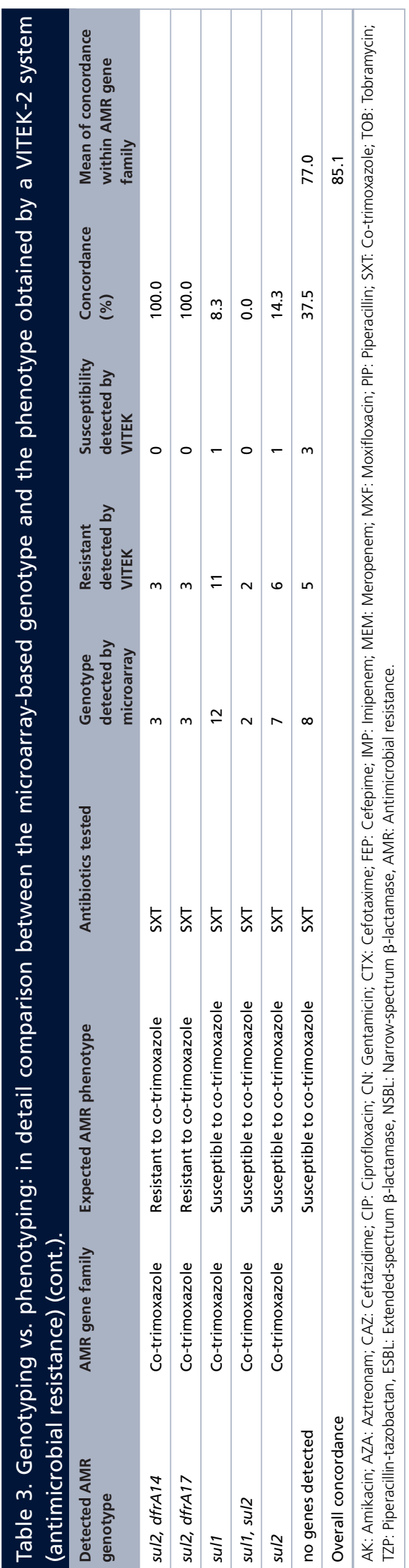


The probes and primers for blaTEM and blaSHV were designed to aim on consensus areas (Supplementary Table 5). Therefore, blaTEM and blaSHV ESBLs cannot be distinguished from non-ESBL blaTEM and blaSHV alleles.

Multiplex labeling by primer extension amplification and hybridization was done according to the manufacturer's instructions. The procedure for multiplex labeling, hybridization and data analysis is described in detail by Braun et al. (2014) [27]. Starting from a clonal overnight culture, the complete turnaround time of the described kit is approximately $7 \mathrm{~h}$, with a hands-on time of approximately $3 \mathrm{~h}$.

\section{Ethics statement}

All isolates were collected from Kidney Center, Al-Sayed Hospital, Rawalpindi, Pakistan. Isolates were obtained during routine diagnostics and were analyzed anonymously. None of the authors were involved in sample collection, or interaction with the patients. Isolates were provided anonymously and no patient data were forwarded to co-authors or contributors not affiliated to the Al-Sayed Hospital, who also had no access to any patient-identifying information. Ethics approval and informed consent were therefore not required by EU regulations on epidemiological surveillance studies.

\section{Results}

\section{Evaluation of the advanced carbapenemase assay}

The functionality of the CarbDetect AS-2 kit was tested using 97 different reference strains (see above) with confirmed AMR-resistance genes. A comparison between all characterized reference strains and the detected AMRgenes is listed in the Table 1. The gene $a a c\left(6^{\prime}\right)-I b-c r$ (strain 278798)-mediating quinolone resistance was false detected as $a a c\left(6^{\prime}\right)-l b$. However, in this strain the confirmed AMR-gene $d f r A 7$ was detected correctly; therefore, the concordance here was 50\% (Table 1). The overall concordance between the detected AMR-genes and the AMR-genes confirmed to be present in the reference strains was $98.8 \%$.

No reference strains were available for blaKHM, blaPAM-1, blaSFH-1, blaSMB-1, blaOXA-279, blaOXA-292, blaFOX, blaMOX, acc(6)-II, aac(6)-IIa, aac-aph, npmA and blaMOX-CMY9. The detailed, complete experimental data for all 97 reference strains are given in the Supplementary Table 1.

\section{Samples \& clinical isolates}

Carbapenem-resistant isolates were found in blood, bronchoalveolar lavage, central venous pressure tube, double-J stent, endotracheal tube, Foley catheter, nasogastric tube, pus, throat swab, urine and wound swab. Escherichia coli was the most prevalent pathogen (Table 2). However, in urine samples $K$. pneumoniae was as often isolated as $E$. coli and on Foley catheters $K$. pneumoniae was the most common species $(\mathrm{n}=7)$. Acinetobacter baumannii was the most frequently found pathogen in pus.

The carbapenemase gene blaNDM was found in nearby all types of samples, except in blood, on the double-J stents and wound swab (Table 2), but this might be attributed to low sample numbers. The gene blaOXA-23 was exclusively detected in A. baumannii.

\section{Susceptibility testing \& species identification of clinical isolates}

Of 425 isolates collected during the period of our study, 82 (19.3\%) were preliminarily identified as carbapenemresistant using disk diffusion assays. According to confirmatory tests of these 82 isolates, 72 were resistant to meropenem and imipenem indeed. Out of the ten carbapenem susceptible isolates, nine were still resistant to cefepime and one against ceftazidim (Supplementary Table 2).

Carbapenem-resistant isolates were identified as K. pneumoniae $(\mathrm{n}=26), E$. coli $(\mathrm{n}=22), P$. aeruginosa $(\mathrm{n}=15)$, A. baumannii $(\mathrm{n}=8)$, E. cloacae $(\mathrm{n}=4)$, Stenotrophomonas maltophilia $(\mathrm{n}=2)$, Achromobacter xylosoxidans $(\mathrm{n}=2)$, Pseudomonas oleovorans $(\mathrm{n}=2)$ and $P$. putida $(\mathrm{n}=1)$.

Eight isolates (K. pneumoniae, $\mathrm{n}=6 ;$ S . maltophilia, $\mathrm{n}=2)(9.8 \%)$ were resistant to colistin.

\section{Antimicrobial resistance genotype \& phenotype}

Carbapenemase genes were detected in 61 out of the 72 carbapenem-resistant isolates. The most prevalent carbapenemase gene was blaNDM $(\mathrm{n}=31)$ followed by blaOXA-181/232 $(\mathrm{n}=15)$, blaVIM $(\mathrm{n}=8)$, blaOXA-23 $(\mathrm{n}=8)$, blaOXA-51-like $(\mathrm{n}=8)$, blaOXA-48-like $(\mathrm{n}=6)$, blaGES $(\mathrm{n}=3)$, blaIMP $(\mathrm{n}=1)$ and blaSIM-1 $(\mathrm{n}=1)$ (Table 2). In 12 isolates, multiple carbapenemase genes were detected. The following combinations were 
found, blaNDM/blaOXA-48-like ( $\mathrm{n}=6)$, blaNDM/blaOXA-181/232 ( $=3)$, blaNDM/blaGES $(\mathrm{n}=2)$ and blaVIM/blaGES $(\mathrm{n}=1)$ (Table 2). In the remaining 11 carbapenem-resistant isolates, no carbapenemase genes were detected (Table 2). Modified Hodge tests also yielded negative results suggesting another mechanism of resistance such as overexpression of AmpC accompanied by a porin loss or efflux pumps. Due to these 11 isolates (Supplementary Table 3), the concordance between genotype and phenotype for carbapenem-resistant isolates was 95.2\% (Table 3). The insertion sequence ISABal was not detected upstream to the intrinsic carbapenemase gene blaOXA-51 in A. baumannii. The underlying mechanism of detected carbapenem-resistant in A. baumannii was blaOXA-23 (Table 2).

Out of 21 isolates with no carbapenem-resistance genes, 17 were still resistant to third and fourth generation cephalosporins (Table 3). In 13 of these 17 isolates, ESBL genes were detected (Table 2). The gene blaCTX-M-1/15 was found in ten of these isolates, two of which harbored bla VEB while one isolate was only positive for consensus marker blaTEM and blaSHV (both including also ESBL variants); thus, the pheno- and genotypes correlated perfectly (Table 3).

In the remaining eight isolates, no ESBL gene was detected, albeit four of them were still resistant to all cephalosporins. These included two S. maltophilia and two $P$. aeruginosa isolates that were resistant to all other $\beta$-lactams. For details see Supplementary Table 3.

All isolates were screened for 28 different aminoglycoside resistance genes. Sixty-nine out of $82(84.1 \%)$ isolates were resistant to at least one tested aminoglycoside (amikacin, tobramycin and/or gentamicin). Thirty-three different genotypes (i.e., combinations of these genes) were identified (Table 3). The most prevalent genotypes were $\operatorname{acc}\left(6^{\prime}\right)-I b(\mathrm{n}=5)$ and $a a c\left(6^{\prime}\right)-I b$ in combination with $\operatorname{add} A 1$, $\operatorname{aad} A 2, \operatorname{arm} A, \operatorname{str} A, \operatorname{str} B(\mathrm{n}=5)$ or $\operatorname{aac}\left(6^{\prime}\right)-I b$ with $\operatorname{add} A 2$ $(\mathrm{n}=5)$ or $\operatorname{aac}\left(6^{\prime}\right)-I b$ with $a a d A 2, \operatorname{str} A, \operatorname{str} B(\mathrm{n}=5)$ or $\operatorname{aac}\left(6^{\prime}\right)-I b$ with $\operatorname{rmt} C, \operatorname{str} A, \operatorname{str} B(\mathrm{n}=5)$. All isolates harboring these genotypes were resistant to all tested aminoglycosides (Table 3 ). The genotype $a a c\left(6^{\prime}\right)$ - $I b$ in combination with aadA4, $\operatorname{str} A, \operatorname{str} B(\mathrm{n}=4)$ showed a $100 \%$ concordance to the detected resistance to all aminoglycosides tested.

Two out of four isolates that harbored only $\operatorname{str} A / \operatorname{str} B$ (mediating resistance to streptomycin only, which was not tested) were also resistant to amikacin, tobramycin and gentamycin. These isolates were found to be resistant to more compounds than expected; therefore the concordance was set to $50 \%$. For isolates carrying only aadA2 $(\mathrm{n}=3)$, which confers resistance only to streptomycin and spectinomycin, the concordance was $100 \%$, because the isolates were susceptible to all tested aminoglycosides. For isolates carrying just aadAl $(\mathrm{n}=3)$, which only confers resistance to streptomycin and spectinomycin, two were still resistant to all tested aminoglycosides, and therefore the concordance was $33.3 \%$. One isolate harbored the combination of aadA4 and $\operatorname{str} B$, and it was susceptible to all aminoglycosides (concordance $0.0 \%$ ). Although streptomycin and spectinomycin were not tested in the recent study, previous studies using the same combination of probes and primers for aadA1 and aadA2 showed that isolates encoding these genes were only resistant to streptomycin and spectinomycin [32].

In eight isolates, no aminoglycoside resistance genes were detected, although two of them were resistant to all aminoglycosides tested. The mean concordance between aminoglycoside resistance genotype and phenotype was 87.9\% (Table 3).

Seventy-six out of $82(92.7 \%)$ isolates were resistant to fluoroquinolones. However, in only 32 isolates corresponding fluoroquinolones resistance genes were detected (qepA, qnrA1, qnrB, qnrS). All isolates that harbored at least one of these genes were resistant to both tested fluoroquinolones (ciprofloxacin, moxifloxacin). For these isolates, geno- and phenotype correlated perfectly. In 44 fluoroquinolone-resistant isolates and in six susceptible isolates, no genes could be found that are associated with fluoroquinolone resistance. Therefore, the mean concordance between fluoroquinolone-resistant phenotype and genotype was $85.3 \%$ (Table 3 ).

Seventy-four out of $82(90.2 \%)$ isolates were resistant to co-trimoxazole. Resistance to co-trimoxazole suggests a genotype of sul in combination with $d f r A$. Most tested isolates (50 of 53) with this gene combination were resistant to co-trimoxazole indeed. Isolates with the following genotypes were exceptions: sul1/dfrA12 (seven of eight isolates were resistant, $87.5 \%$ concordance); sul1/dfrA5/dfrA12 (isolate was susceptible, $0.0 \%$ concordance), sul1/sul2/dfrA12 (isolate was susceptible, $0.0 \%$ concordance). Of 12 isolates that harbored only the gene sul1, 11 were still resistant (concordance 8.3\%) and all isolates with the genotype sul1/sul2 were resistant (concordance $0.0 \%$ ). Six of seven isolates where only the gene sul 2 was detected were resistant (concordance $14.3 \%$ ). Five of eight isolates harbored no gene for co-trimoxazole resistance but were still found to be resistant (concordance 37.5\%). Therefore, the mean concordance of genotype and phenotype was $77 \%$ (Table 3 ).

Eight out of $82(9.8 \%)$ isolates were colistin resistant, but none of these isolates harbored the genes $m c r-1$ or mor-2. 
The overall concordance among all genotypes to expected phenotypes was $85.1 \%$ (Table 3).

\section{Discussion}

We report the prevalence of carbapenemase-producing Gram-negative pathogens at the Kidney Center, Rawalpindi, Pakistan. All presented genotyping data were determined using the CarbDetect AS-2 kit, which is an updated version of the CarbDetect AS-1 kit [27]. This update includes not only additional markers for carbapenemase genes, but also markers for resistance mechanisms toward other classes of antibiotics (i.e., aminoglycosides, fluoroquinolones and others). Evaluation with 97 defined reference strains showed a 98.8\% concordance between expected and detected genotypes. No reference strains were available for some rare resistance genes (see above); in these cases an evaluation was not possible.

Since blaNDM-1 was first reported from India in 2009, it is believed that CPOs are highly prevalent in this region and are disseminating globally from there [11,33-37]. The current worldwide emergence of resistance to carbapenems in Gram-negative pathogens constitutes an important and growing public health threat. Sporadic outbreaks of carbapenem-resistant organisms have been reported not only in hospitals, but also in the community [26. In this study, the prevalence of carbapenem-resistance isolates was about $1 \%$ of all clinical samples collected during the period of this study $(\mathrm{n}=7857)$, but $19.3 \%$ of all samples from which clinically relevant Gram-negatives were cultured $(\mathrm{n}=425)$. Our findings were in accordance to a previous study at a military hospital in Rawalpindi, Pakistan. Carbapenemase-producing Enterobacteriaceae were detected in 32 out of 175 isolates (18.3\%) from stool samples and all produced blaNDM carbapenemase [13].

In the present study, 82 of 425 isolates were initially identified as carbapenem resistant using disc diffusion tests. However, confirmatory tests using VITEK-2 system revealed that in fact 72 samples were resistant. Carbapenemase genes were detected in 61 isolates. For the remaining 11 isolates in which no carbapenemase genes were detected, negative modified Hodge tests (MHT) indicated that the resistance mechanisms were an AmpC overexpression in combination with porin loss [28,30] or a different mechanism. For $P$. aeruginosa, the carbapenem-resistance is usually mediated by OprD loss, which primarily confers a resistance to imipenem but also confers a low-grade resistance to meropenem [38]. Stenotrophomonas maltophilia is resistant to all $\beta$-lactams because of two native and ubiquitous broad-spectrum metallo- $\beta$-lactamases (L1/L2) [31] that are not covered by the assay used herein. It is well known that MHT lacks specificity and may produce false-positive results for bacteria with complex ESBL or AmpC backgrounds [39]. In the recent study all carbapenem-resistant clinical isolates with at least one detected carbapenemase gene were positive using MHT. All carbapenem-resistant isolates lacking carbapenemase genes were negative. All used methods (VITEK, MHT and microarray) correlated perfectly. Furthermore, to the best of our knowledge, up-to-date false-negative MHTs were not described.

While blaKPC [40] is the most prevalent carbapenemase gene globally, blaNDM proved to be the most common carbapenemase gene in the study region, and it was detected in almost $50 \%$ of all carbapenemase-producing isolates belonging to different species in this study. Previous studies from Pakistan have reported variable prevalence for blaNDM. One study at a military hospital reported that blaNDM was the only carbapenemase resistance gene detected in their samples [13]. In 2010, Kumarasamy et al. also reported blaNDM-1 in Pakistan [11]. In their study, the gene encoding NDM was the most frequently found carbapenemase and it was detected in nearly all sample types. Another study reported 18.5\% prevalence of blaNDM-1-positive Enterobacteriaceae isolated from 200 stool samples [14]. Additionally, Nahid et al. indicated 23\% prevalence of blaNDM-1 gene, 25\% blaVIM gene and 1.5\% blaIMP gene [4]. Sattar et al. documented a prevalence of blaNDM of $28.8 \%$ in P. aeruginosa, $14.6 \%$ in E. coli and $5.0 \%$ in K. pneumonia [41]. blaNDM-1 $\beta$-lactamase-producing bacteria are detected not only in hospital settings, but also in environmental samples (drinking water and seepage water). In New Delhi, blaNDM-1 was detected in two out of 50 drinking water samples and in 51 out of 171 seepage samples [42].

The second most prevalent enzyme in the current study was blaOXA-181/232 followed by blaVIM. However, blaOXA-23 was detected in only $A$. baumanii isolates. Irfan $e$ al. collected carbapenem-resistant $A$. baumannii (CRAB) samples from a tertiary care hospital in Karachi and found blaOXA-23-like acquired-oxacillinase genes in 47 out of 50 isolates [43]. In another study, blaOXA-23 was most commonly associated with resistance among 125 CRAB isolates [44]. Similar results were also found by Atrouni et al. [45]. Another study reported blaOXA-23 in 14 out of 59 CRAB isolates [35]. In the current study, blaOXA-23 was the only carbapenemase gene identified in all eight CRAB isolates.

The treatment of carbapenem-resistant pathogens is very challenging and colistin is commonly used to treat such patients. Colistin is an old antibiotic that has largely been avoided during the last decades due to its toxicity. 
Nonetheless, it is one of the few agents that remain effective against multidrug-resistant Gram-negative bacteria, resulting in an increasing clinical usefulness for colistin [46]. Colistin resistance is normally mediated due to mutations affecting chromosomal genes and not associated with horizontal gene transfer. However, in PR China, a plasmidencoded colistin-resistance gene, $m c r-1$, was recently identified in colistin-resistant $E$. coli, isolated particularly from swine [19]. Recently, a second variant of a colistin resistance gene named $m c r-2$ was detected in E. coli also from swine [47]. In our study, $10 \%$ of all isolates were colistin resistant, but none of the isolates harbor the gene $m c r-1$ and $m c r-2$ genes, so we assume that all detected resistance to polymyxin was chromosomally mediated, that is, due to mutations and possible favored by previous episodes of colistin treatment of these individual patients. Unfortunately, data on prior antibiotic prescriptions were not accessible.

Combined resistance to various antibiotics was also evaluated in the present study and an overall $89 \%$ resistance to fluoroquinolones was observed in carbapenem-resistant isolates. Zhang et al. conducted a study in PR China and they reported 93\% fluoroquinolone resistance in carbapenem-resistant E. coli strains [48]. Sekyere et al. (2017), recently reported of a higher fluoroquinolone, tigecycline and colistin resistance among carbapenemase-producing Enterobacteriaceae isolates from South Africa [49,50].

Interestingly, 12 isolates were shown to harbor multiple carbapenemase genes (Table 2). These isolates carried blaNDM in combination with blaOXA-48-like genes $(\mathrm{n}=9)$ or blaGES $(\mathrm{n}=2)$; or blaVIM in combination with blaGES $(\mathrm{n}=1)$. There has been a recent report from south India on the occurrence of oxacillinase production that also demonstrated predominant presence of blaVIM along with blaOXA-23 among clinical isolates [51]. Although a report from south India has investigated co-presence of blaOXA-23 with blaNDM-1 [52], this is the first study from Pakistan which has detected multiple genetic mechanisms, blaNDM, blaVIM, different blaOXA-48-like genes and blaGES in different species (K. pneumoniae, A. xylosoxidans, P. oleovorans). We hypothesize that this co-existence of multiple resistance genes is due to the presence of multiple resistance plasmids in the isolates. This hypothesis will be supported by the assumption that the acquisition of plasmids carrying resistance genes may not impose a substantial cost to the recipient bacteria [53-56]. We assume that antibiotic selective pressure is the driving force to include more than one carbapenemase genes. Maybe the overuse of different carbapenems and other antibiotics in hospitals selected these pathogens which are resistant to multiple antibiotics.

In this context, the multiplex microarray method used in this study is well suited for analyzing bacteria which are multidrug resistant. Carbapenemase and other genes encoding for resistance to aminoglycosides, fluoroquinolones and co-trimoxazole can also be detected with a single reaction. Genes coding for narrow-spectrum $\beta$-lactamases, AmpC and ESBLs will be detected simultaneously.

We are aware that next-generation sequencing (NGS) is also suitable for multiparameter testing, but microarray platforms have a proven track record spanning nearly two decades in the lab. Researchers worldwide are familiar with both operating the technology and analyzing the results. Microarrays are easier to use with less complicated and less labor-intensive sample preparation than NGS [57]. The same goes for data analysis. Despite the rapid drop in the cost associated with NGS arrays are still more economical and yield higher throughput, providing significant advantages when working with a large number of samples.

\section{Conclusion}

Out of 425 Gram-negative isolates, 72 (17\%) were resistant to imipenem and meropenem, and in 61 (14\%) cases carbapenemase genes were detected. All isolates with at least one carbapenemase gene were resistant against tested carbapenems. The comparison of the genotyping to phenotyping profiles for each target should rather be used to determine to what extent or not the detection of a gene leads to the expected functionality. This because all molecular rapid tests that detect DNA directly from a real sample without previous culture work on the assumption that a gene is always translated into the corresponding function. In the end, the phenotypic resistance is crucial for the clinician and diagnostician when he has to make an appropriate therapy decision. Therefore, these data are of considerable relevance for developers of molecular point of care tests.

Due to the high concordance between the genotypic and phenotypic profiles, we assume that the detection of the genotype gives a very good indication of the distinct phenotype. Therefore, a fast genetic analysis that could detect different resistance genes simultaneously, like the array-based method described in this paper, could be used to detect patients harboring these multiresistance bacteria. Genotyping results may not be used for treatment decisions, but to make a decision whether to isolate a positive patient, and decide the next logical diagnostic procedures for the bacteria. 
Furthermore, antimicrobial stewardship has been suggested as a significant way forward to control MDR pathogens especially for CPO pathogens. Therefore, fast detection of responsible genes for ESBL and/or carbapenem resistance could be useful to control the overuse of antibiotics.

\section{Future perspective}

One of the main challenges of our time is the dramatic spread of pathogenic microorganisms that are resistant toward antibiotics, such as carbapenem-resistant Enterobacteriaceae (CRE) and MRSA. Recent global trends like constant economic growth, globalization, industrial agriculture, farming and food production, outsourcing and centralization of pharmaceutical production of antimicrobial substances, mass transport of livestock, worldwide travel and migration, aging societies combined with modern medicine, false and nondirected use of antibiotics, lack of hygiene, insufficient availability of safe food and clean drinking water, anti-vaxxer movements, insufficient epidemiological monitoring, poor patient to staff ratios in rehabilitation centers, hospitals, and care facilities, as well as delayed diagnosis combined with the economization of medical procedures are main drivers to antibiotic resistance, and this trend will accelerate in the future. This also affects Pakistan. To stop and to reverse these developments, global and trans-sectoral action is urgently needed. One out of many important challenges is the global availability of decentralized (point of care) diagnostics that can guide therapy in order to avoid an unnecessary use of antimicrobial substances.

\section{Summary points}

The medical need

- Misidentification of carbapenem resistance could lead to inappropriate antibiotic treatment and dissemination of resistance.

- An urgent need exists for diagnostic laboratories to introduce methods for a rapid molecular identification of carbapenemase and extended-spectrum $\beta$-lactamases genes.

- Carbapenem resistance appears to be common in Pakistan. Therefore, we characterized carbapenemase-producing organisms from a tertiary care center in Rawalpindi, Pakistan using a DNA microarray. The microarray technique

- The microarray-based assay presented herein provides a new option to simultaneously identify 43 globally important carbapenemase genes and their allelic variants starting from a clonal culture fast and economically.

- Comparing genotypic to phenotypic data, their correlation indicates whether a genotype can be used to develop rapid tests or can be used directly in hospital settings and epidemiological investigations.

Evaluation of the microarray-based assay

- The herein described DNA-based antimicrobial resistance (AMR) assay was evaluated using defined reference strains.

- Clinical isolates from the Kidney Center of Rawalpindi, Pakistan were used to compare the DNA microarray-detected genotype with the corresponding phenotype (analyzed by VITEK-2).

Outcome

- The overall concordance between expected and detected genotypes using 97 defined reference strains was $98.8 \%$

- The overall concordance between genotyping and phenotyping profile was over $85 \%$.

- The detection of the genotype gives a very good indication of the distinct phenotype.

- During routine diagnostics of 7857 human samples $425 \mathrm{Gram}$-negative isolates were tested for resistance to carbapenems.

- Seventy-two of four hundred and twenty-five (17\%) were resistant to imipenem and meropenem, and in 61 $(14 \%)$ cases carbapenemase genes were detected.

- The most prevalent carbapenemase gene was blaNDM $(n=31)$.

Conclusion

- The current DNA-based AMR assay could be used to control multidrug-resistant pathogens especially carbapenemase-producing organism.

- Our study demonstrates that the detection of the AMR genotype gives a very good indication of the distinct AMR phenotype.

- The data presented in this study is of considerable relevance for developers of molecular point of care tests. 


\section{Acknowledgments}

The authors are thankful to the kidney center at the Al-Sayed Hospital, Rawalpindi, Pakistan for providing microbial samples for this research.

Financial \& competing interests disclosure

This study was supported by the Office of the Vice Chancellor and the Office of Research, Innovation and Commercialization of the University of Haripur, Haripur, Pakistan. The authors have no other relevant affiliations or financial involvement with any organization or entity with a financial interest in or financial conflict with the subject matter or materials discussed in the manuscript apart from those disclosed. No writing assistance was utilized in the production of this manuscript.

\section{Supplementary data}

To view the supplementary data that accompany this paper please visit the journal website at: www.futuremedicine.com/doi/full/10.2217/fmb-2018-0082

\section{Open access}

This work is licensed under the Attribution-NonCommercial-NoDerivatives 4.0 Unported License. To view a copy of this license, visit http://creativecommons.org/licenses/by-nc-nd/4.0/

\section{References}

Papers of special note have been highlighted as: $\bullet$ of interest; $\bullet \bullet$ of considerable interest

1. Rupp ME, Fey PD. Extended spectrum $\beta$-lactamase (ESBL)-producing Enterobacteriaceae: considerations for diagnosis, prevention and drug treatment. Drugs 63(4), 353-365 (2003).

2. Al-Assil B, Mahfoud M, Hamzeh AR. Resistance trends and risk factors of extended spectrum $\beta$ beta-lactamases in Escherichia coli infections in Aleppo, Syria. Am. J. Infect. Control. 41(7), 597-600 (2013).

3. Brandt $\mathrm{C}$, Braun SD, Stein $\mathrm{C}$ et al. In silico serine $\beta$-lactamases analysis reveals a huge potential resistome in environmental and pathogenic species. Sci. Rep. 7, 1-13 (2017).

-. This excellent review is an example of the fact that we have reached only the tip of the iceberg in relation to known resistance genes. It shows the dramatic diversity of $\beta$-lactamases as an underestimated reservoir in environmental bacteria. These bacteria represent an uncharacterized reservoir of potentially transferable resistance genes.

4. Nahid F, Khan AA, Rehman S, Zahra R. Prevalence of metallo- $\beta$-lactamase NDM-1-producing multi-drug resistant bacteria at two Pakistani hospitals and implications for public health. J. Infect. Public Health 6(6), 487-493 (2013).

- This paper shows the high prevalence of blaNDM in Pakistan. The described numbers are in concordance with our findings in the current paper.

5. Hrabak J, Chudackova E, Papagiannitsis CC. Detection of carbapenemases in Enterobacteriaceae: a challenge for diagnostic microbiological laboratories. Clin. Microbiol. Infect. 20(9), 839-853 (2014).

6. Jamil B. Enterobacteriaceae: At the verge of treatment. Int. J. Inn. Appl. Stud. 9(4), 1736-1745 (2014).

7. Crandon JL, Nicolau DP. Human simulated studies of aztreonam and aztreonam-avibactam to evaluate activity against challenging gram-negative organisms, including metallo- $\beta$-lactamase producers. Antimicrob. Agents Chemother. 57(7), 3299-3306 (2013).

8. Patel G, Huprikar S, Factor SH, Jenkins SG, Calfee DP. Outcomes of carbapenem-resistant Klebsiella pneumoniae infection and the impact of antimicrobial and adjunctive therapies. Infect. Control Hosp. Epidemiol. 29(12), 1099-1106 (2008).

9. Queenan AM, Bush K. Carbapenemases: the versatile $\beta$-lactamases. Clin. Microbiol. Rev. 20(3), 440-458 (2007).

10. Schwaber MJ, Klarfeld-Lidji S, Navon-Venezia S, Schwartz D, Leavitt A, Carmeli Y. Predictors of carbapenem-resistant Klebsiella pneumoniae acquisition among hospitalized adults and effect of acquisition on mortality. Antimicrob. Agents Chemother. 52(3), 1028-1033 (2008).

11. Kumarasamy KK, Toleman MA, Walsh TR et al. Emergence of a new antibiotic resistance mechanism in India, Pakistan, and the UK: a molecular, biological, and epidemiological study. Lancet Infect. Dis. 10(9), 597-602 (2010).

-• Herein, the authors investigate the prevalence of blaNDM-1, in multidrug-resistant Enterobacteriaceae in India, Pakistan and the UK. As also observed by us, blaNDM was the most prevalent carbapenemase gene in Escherichia coli and Klebsiella pneumoniae.

12. Bushnell G, Mitrani-Gold F, Mundy LM. Emergence of New Delhi metallo- $\beta$-lactamase type 1-producing Enterobacteriaceae and non-Enterobacteriaceae: global case detection and bacterial surveillance. Int. J. Infect. Dis. 17(5), e325-e333 (2013).

13. Day KM, Ali S, Mirza IA et al. Prevalence and molecular characterization of Enterobacteriaceae producing NDM-1 carbapenemase at a military hospital in Pakistan and evaluation of two chromogenic media. Diagn. Microbiol. Infect. Dis. 75(2), 187-191 (2013). 
14. Perry JD, Naqvi SH, Mirza IA et al. Prevalence of faecal carriage of Enterobacteriaceae with NDM-1 carbapenemase at military hospitals in Pakistan, and evaluation of two chromogenic media. J. Antimicrob. Chemother. 66(10), 2288-2294 (2011).

-• An important study for us as it was also carried out in Rawalpindi, Pakistan, albeit in other hospitals. Shows a high prevalence of multidrug-resistant Enterobacteriaceae carrying the blaNDM-1 gene. Multiresistant bacteria were isolated from stool samples of both patients and colonized outpatients, indicating a high prevalence also among colonized carriers in the community.

15. Morrill HJ, Pogue JM, Kaye KS, Laplante KL. Treatment options for carbapenem-resistant Enterobacteriaceae infections. Open Forum Infect. Dis. 2(2), ofv050 (2015).

16. Van Duin D, Cober ED, Richter SS et al. Tigecycline therapy for carbapenem-resistant Klebsiella pneumoniae (CRKP) bacteriuria leads to tigecycline resistance. Clin. Microbiol. Infect. 20(12), O1117-O1120 (2014).

17. Kempf I, Fleury MA, Drider D et al. What do we know about resistance to colistin in Enterobacteriaceae in avian and pig production in Europe? Int. J. Antimicrob. Agents 42(5), 379-383 (2013).

18. Cortegiani A, Russotto V, Graziano G et al. Use of Cepheid Xpert Carba-R(R) for rapid detection of carbapenemase-producing bacteria in abdominal septic patients admitted to intensive care unit. PLoS ONE 11(8), e0160643 (2016).

19. Liu YY, Wang Y, Walsh TR et al. Emergence of plasmid-mediated colistin resistance mechanism MCR-1 in animals and human beings in China: a microbiological and molecular biological study. Lancet Infect. Dis. 16(2), 161-168 (2016).

20. Cunha CB, Kassakian SZ, Chan R et al. Screening of nursing home residents for colonization with carbapenem-resistant Enterobacteriaceae admitted to acute care hospitals: incidence and risk factors. Am. J. Infect. Control. 44(2), 126-130 (2016).

21. Lledo W, Hernandez M, Lopez E et al. Guidance for control of infections with carbapenem-resistant or carbapenemase-producing Enterobacteriaceae in acute care facilities. MMWR Morb. Mortal. Wkly Rep. 58(10), 256-260 (2009).

- This report from the USA provides recommendations for the control of CRE or carbapenemase-producing Enterobacteriaceae in acute care facilities. An aggressive infection control strategy for all acute care facilities including managing all patients with CRE using contact precautions should be implemented. Also, special guidelines for detection of carbapenemase-producing organism according to the Clinical and Laboratory Standards Institute should be installed.

22. Kallen MD, Ricks P, Edwards J et al. Vital signs: carbapenem-resistant Enterobacteriaceae. MMWR Morb. Mortal. Wkly Rep. 62(9), 165-170 (2013).

23. Vogne C, Prod'hom G, Jaton K, Decosterd LA, Greub G. A simple, robust and rapid approach to detect carbapenemases in Gram-negative isolates by MALDI-TOF mass spectrometry: validation with triple quadripole tandem mass spectrometry, microarray and PCR. Clin. Microbiol. Infect. 20(12), O1106-O1112 (2014).

24. Huang TD, Bogaerts P, Ghilani E et al. Multicentre evaluation of the Check-Direct $\mathrm{CPE}(\mathrm{R})$ assay for direct screening of carbapenemase-producing Enterobacteriaceae from rectal swabs. J. Antimicrob. Chemother. 70(6), 1669-1673 (2015).

25. Boran N, Vivian B, Logan C, Grogan J. Formation of a carbapenemase resistance detection algorithm for use in the routine laboratory. Br. J. Biomed. Sci. 72(1), 12-22 (2015).

26. Nordmann P, Dortet L, Poirel L. Carbapenem resistance in Enterobacteriaceae: here is the storm! Trends Mol. Med. 18(5), 263-272 (2012).

27. Braun SD, Monecke S, Thurmer A et al. Rapid identification of carbapenemase genes in Gram-negative bacteria with an oligonucleotide microarray-based assay. PLoS ONE 9(7), e102232 (2014).

-• Reports in detail the previous version of the CarbaDetrect AS-2 Kit. Herein, the microarray (design, platform), all methods (labeling, hybridization, staining) and the automatic analysis software are described.

28. Carvalhaes CG, Picao RC, Nicoletti AG, Xavier DE, Gales AC. Cloverleaf test (modified Hodge test) for detecting carbapenemase production in Klebsiella pneumoniae: be aware of false positive results. J. Antimicrob. Chemother. 65(2), 249-251 (2009).

29. Doumith M, Ellington MJ, Livermore DM, Woodford N. Molecular mechanisms disrupting porin expression in ertapenem-resistant Klebsiella and Enterobacter spp. clinical isolates from the UK. J. Antimicrob. Chemother. 63(4), 659-667 (2009).

30. Martinez-Martinez L, Pascual A, Hernandez-Alles $S$ et al. Roles of $\beta$-lactamases and porins in activities of carbapenems and cephalosporins against Klebsiella pneumoniae. Antimicrob. Agents Chemother. 43(7), 1669-1673 (1999).

31. Osei Sekyere J, Govinden U, Bester LA, Essack SY. Colistin and tigecycline resistance in carbapenemase-producing Gram-negative bacteria: emerging resistance mechanisms and detection methods. J. Appl. Microbiol. 121(3), 601-617 (2016).

32. Braun SD, Ziegler A, Methner U et al. Fast DNA serotyping and antimicrobial resistance gene determination of Salmonella enterica with an oligonucleotide microarray-based assay. PLoS ONE 7(10), e46489 (2012).

33. Begum S, Hasan F, Hussain S, Ali Shah A. Prevalence of multi drug resistant Acinetobacter baumannii in the clinical samples from Tertiary Care Hospital in Islamabad, Pakistan. Pak. J. Med. Sci. 29(5), 1253-1258 (2013).

34. Castanheira M, Deshpande LM, Mathai D, Bell JM, Jones RN, Mendes RE. Early dissemination of NDM-1- and OXA-181-producing Enterobacteriaceae in Indian hospitals: report from the SENTRY Antimicrobial Surveillance Program, 2006-2007. Antimicrob. Agents Chemother. 55(3), 1274-1278 (2011).

35. Hasan B, Perveen K, Olsen B, Zahra R. Emergence of carbapenem-resistant Acinetobacter baumannii in hospitals in Pakistan. J. Med. Microbiol. 63(Pt 1), 50-55 (2014). 
36. Pesesky MW, Hussain T, Wallace M et al. KPC and NDM-1 genes in related Enterobacteriaceae strains and plasmids from Pakistan and the United States. Emerg. Infect. Dis. 21(6), 1034-1037 (2015).

37. Yong D, Toleman MA, Giske CG et al. Characterization of a new metallo- $\beta$-lactamase gene, bla(NDM-1), and a novel erythromycin esterase gene carried on a unique genetic structure in Klebsiella pneumoniae sequence type 14 from India. Antimicrob. Agents Chemother. 53(12), 5046-5054 (2009).

38. Pai H, Kim J, Lee JH, Choe KW, Gotoh N. Carbapenem resistance mechanisms in Pseudomonas aeruginosa clinical isolates. Antimicrob. Agents Chemother. 45(2), 480-484 (2001).

39. Viau R, Frank KM, Jacobs MR et al. Intestinal carriage of carbapenemase-producing organisms: current status of surveillance methods. Clin. Microbiol. Rev. 29(1), 1-27 (2016).

40. Nordmann P, Naas T, Poirel L. Global spread of carbapenemase-producing Enterobacteriaceae. Emerg. Infect. Dis. 17(10), 1791-1798 (2011).

41. Sattar H, Toleman M, Nahid F, Zahra R. Co-existence of blaNDM-1 and blaKPC-2 in clinical isolates of Klebsiella pneumoniae from Pakistan. J. Chemother. 28(4), 346-349 (2016).

- The brief communication is of special interest as it describes also the co-existence of two carbapenemase genes in one organism. The combination of blaNDM and blaKPC was detected in Klebsiella pneumoniae.

42. Walsh TR, Weeks J, Livermore DM, Toleman MA. Dissemination of NDM-1 positive bacteria in the New Delhi environment and its implications for human health: an environmental point prevalence study. Lancet Infect. Dis. 11(5), 355-362 (2011).

43. Irfan S, Turton JF, Mehraj J et al. Molecular and epidemiological characterisation of clinical isolates of carbapenem-resistant Acinetobacter baumannii from public and private sector intensive care units in Karachi, Pakistan. J. Hosp. Infect. 78(2), 143-148 (2011).

44. Selasi GN, Nicholas A, Jeon $\mathrm{H}$ et al. Genetic basis of antimicrobial resistance and clonal dynamics of carbapenem-resistant Acinetobacter baumannii sequence type 191 in a Korean hospital. Infect. Genet. Evol. 36, 1-7 (2015).

45. Atrouni AA, Hamze M, Jisr T et al. Wide spread of Oxa-23 producing carbapenem resistant Acinetobacter baumannii belonging to the clonal complex II in different hospitals in Lebanon. Int. J. Infect. Dis. 52, $29-36$ (2016).

46. Falagas ME, Grammatikos AP, Michalopoulos A. Potential of old-generation antibiotics to address current need for new antibiotics. Expert Rev. Anti-Infect. Ther. 6(5), 593-600 (2008).

47. Xavier BB, Lammens C, Ruhal R et al. Identification of a novel plasmid-mediated colistin-resistance gene, mor-2, in Escherichia coli, Belgium, June 2016. Euro Surveill. doi:10.2807/1560-7917.ES.2016.21.27.30280 (2016).

48. Zhang C, Xu X, Pu S et al. Characterization of carbapenemases, extended spectrum $\beta$-lactamases, quinolone resistance and aminoglycoside resistance determinants in carbapenem-non-susceptible Escherichia coli from a teaching hospital in Chongqing, Southwest China. Infect. Genet. Evol. 27, 271-276 (2014).

49. Osei Sekyere J, Amoako DG. Genomic and phenotypic characterisation of fluoroquinolone resistance mechanisms in Enterobacteriaceae in Durban, South Africa. PLoS ONE 12(6), e0178888 (2017).

50. Osei Sekyere J, Amoako DG. Carbonyl cyanide m-chlorophenylhydrazine (CCCP) reverses resistance to colistin, but not to carbapenems and tigecycline in multidrug-resistant Enterobacteriaceae. Front. Microbiol. 8228 (2017).

51. Amudhan SM, Sekar U, Arunagiri K, Sekar B. OXA $\beta$-lactamase-mediated carbapenem resistance in Acinetobacter baumannii. Indian J. Med. Microbiol. 29(3), 269-274 (2011).

52. Karthikeyan K, Thirunarayan MA, Krishnan P. Coexistence of blaOXA-23 with blaNDM-1 and armA in clinical isolates of Acinetobacter baumannii from India. J. Antimicrob. Chemother. 65(10), 2253-2254 (2010).

- Describes the co-existence of blaNDM-1 and blaOXA-23 in Acinetobacter baumannii. The co-existence of oxacillinase and metallo- $\beta$-lactamase genes results in true multiresistance. The emergence of these powerful co-existent resistance mechanisms as also described in the present study - will seriously limit future therapeutic options.

53. Mongkolrattanothai K, Pumfrey L, Mankin P, Gray BM. Acquisition of high-level mupirocin resistance and its fitness cost among methicillin-resistant Staphylococcus aureus strains with low-level mupirocin resistance. J. Clin. Microbiol. 47(12), 4158-4160 (2009).

54. Bottery MJ, Wood AJ, Brockhurst MA. Adaptive modulation of antibiotic resistance through intragenomic coevolution. Nat. Ecol. Evol. 1(9), 1364-1369 (2017).

55. Hall JPJ, Williams D, Paterson S, Harrison E, Brockhurst MA. Positive selection inhibits gene mobilisation and transfer in soil bacterial communities. Nat. Ecol. Evol. 1(9), 1348-1353 (2017).

56. Loftie-Eaton W, Bashford $\mathrm{K}$, Quinn $\mathrm{H}$ et al. Compensatory mutations improve general permissiveness to antibiotic resistance plasmids. Nat. Ecol. Evol. 1(9), 1354-1363 (2017).

57. Michael DunneW Jr, Pouseele H, Monecke S, Ehricht R, Van Belkum A. Epidemiology of transmissible diseases: array hybridization and next generation sequencing as universal nucleic acid-mediated typing tools. Infect. Genet. Evol. 63, 332 -345 (2017). 